\title{
Statistical Stability of Ultrawideband Time-Reversal Imaging in Random Media
}

\author{
Ahmed E. Fouda and Fernando L. Teixeira
}

\begin{abstract}
We carry out a study on the statistical stability of ultrawideband (UWB) time-reversal (TR) imaging in random media under different combinations of random medium parameters and interrogating signal properties. We examine conditions under which frequency decorrelation in random media provides a more effective "self-averaging" and therefore better statistical stability. We also present a new frequency-synthesized technique for UWB time-reversal-based imaging. This technique is employed to construct time-reversal-operator-decomposition (DORT under its French acronym) and multiple-signal-classification (MUSIC) images using either linear or full-aspect transceiver array configurations. The proposed technique automatically provides the best images of desired target(s) in terms of focusing resolution without the need for (synthetic) propagation of time-reversed signals and ad hoc determination of the optimal focusing time instant. In addition, information about the imaging domain (background) can be stored and reused to reconstruct different targets.
\end{abstract}

Index Terms-Frequency decorrelation, MUltiple SIgnals Classification (MUSIC), statistical stability, time-reversal, volumetric beamforming.

\section{INTRODUCTION}

$\mathbf{E}$ VER since capabilities of time-reversal (TR) techniques were first illustrated by Fink and collaborators [1] for acoustic (scalar) waves, considerable theoretical and experimental effort has been put into their extension to electromagnetic and elastic waves and their use to augment detection and imaging algorithms in rich-scattering environments. TR techniques are based on the invariance of the wave equation under time reversal (in lossless reciprocal media). When waves emanated from a source (or reflected by a passive scatterer) are recorded by a sensor array, time-reversed, and retransmitted (in a first-in last-out fashion) in the same medium, they will automatically retrace their "paths" and focus at the (original) source/scatterer location, where the field adds coherently throughout the frequency band of operation.

Backpropagation of time-reversed waves can take place either in the original physical medium (physical TR) or in a synthetic imaging medium (synthetic TR). The former approach finds applications in areas such as lithotripsy [2], and wireless communications [3]. Synthetic TR, on the other hand, is typically used for detection and imaging of targets [4], [5], [6], [7], [8], [9], [10], [11], with applications in ground penetrating radar [4], [5], through-wall imaging [6], and medical imaging [10], for example. A combination of both physical and synthetic TR has been recently applied for detection and tracking of moving targets [12].

The authors are with the ElectroScience Lab., the Department of Electrical and Computer Engineering, The Ohio State University, Columbus, OH USA e-mail:fouda.1@osu.edu, teixeira@ece.osu.edu

July 2011
TR in rich-scattering environments exhibits the distinctive property of superresolution. This means the ability to overcome the classical diffraction limit, enabled by multipathing [13], [14]. Another attractive property enabled by TR is statistical stability. This feature is of particular importance for imaging targets using synthetic TR in random media and for physical TR in scenarios involving time-varying media such as dust, smoke, and/or vegetation. Under certain conditions, "stable" images can be constructed that depend only on the statistical properties of the random medium, and not on the particular realization [15], [16]. Statistical stability of TR was studied in the past [17], [15], [18], [16], where it was shown that physical TR from spatially distributed sources/targets is stable given a sufficiently wideband operation. The performance of TR in time-varying media (where there is a mismatch between the background medium used for the forward problem and the one used for TR backpropagation) was considered in [19], [20]. In this paper, we investigate the statistical stability of TR-based imaging in continuous random media. In this case, mismatch between forward and imaging backgrounds occurs because the (random medium) realization where forward propagation has occurred is not known exactly. A TR-based signal-processing technique that is often used when multiple targets are present is the time-reversal operator decomposition (DORT, under its French acronym) [21], [22], [23], [24], [25]. DORT is based upon an eigenspace analysis of the timereversal operator (TRO). If the scatterers are well-resolved by the TR array, eigenvectors in the signal subspace of the TRO can be used to yield selective focusing (beamforming) on each scatterer individually. Another effective subspace technique for TR applications is the Multiple SIgnal Classification (MUSIC) algorithm, which can be applied on the null subspace to obtain a collective image of all targets.

This paper is divided into two main parts. First, we present a new frequency-synthesized algorithm for constructing UWB TR images. Conventional TR images are typically obtained by backpropagating time-reversed wavefields in a synthetic imaging domain and capturing the image at an appropriate time reference " $t=0$ ", associated with the assumed instant when the target has initiated signal transmission (active target/passive radar mode) or the assumed two-way travel time (passive target/active radar mode) [25]. However, the " $t=0$ " reference may not always be known precisely and/or may not even be the optimal focusing instant. For example, if the (assumed) imaging domain properties do not correspond to those of the physical medium (for example, different mean permittivities and hence different travel-times), the optimal focusing instant will be unknown. The proposed algorithm avoids this problem by employing a spatiotemporal peak 
search to automatically determine both the focusing location and optimal time instant (of focusing). This algorithm is very efficient in terms of processing time, and information about the imaging domain can be stored and reused to construct images of different targets (in a fixed domain). We start by presenting the algorithm in connection to DORT and MUSIC imaging of a point-like scatterer embedded in a homogeneous background using a linear TR array. We compare the resulting DORT and MUSIC images and consider the effect of interrogating signal parameters (center frequency and bandwidth) on the image quality. Next, we apply the same approach to fullaspect arrays, where we introduce "volumetric" beamforming and "volumetric" MUSIC techniques.

In the second part of the paper, we study the statistical stability of time-reversal imaging in continuous random media. In this case, frequency components that constitute the image and which are sufficiently apart can become "incoherent", in the sense of having decorrelated amplitude and phase relationships among them. Under UWB operation, this "frequency decorrelation" leads to self-averaging in the time domain and therefore statistically stable images. We carry out a parametric study on statistical stability of time-reversal imaging with respect to (i) interrogating signal properties (center frequency and bandwidth), (ii) random medium parameters (permittivity variance and correlation length), and (iii) sensor array size and spatial deployment (full- or partial-aspect).

\section{FREQUENCY-SYNTHESIZED IMAGES}

\section{A. Frequency-Synthesized DORT}

To obtain selective imaging of a scene comprising multiple targets/scatterers, eigenstructure (or subspace) methods such as DORT and/or MUSIC can be applied. In this case, a TR transceiver array of $N$ elements is used to produce an $N \times N$ multistatic data matrix (MDM) $\boldsymbol{K}(t)$, where the matrix element $[\boldsymbol{K}(t)]_{i j}$ corresponds to the scattered field (timedomain waveform) recorded by element $i$ from the individual excitation of element $j$ [26]. A Fourier transformation can be applied to obtain a set of MDMs $\boldsymbol{K}(\omega)$ at a discrete set of frequencies within the bandwidth of operation. The timereversal operator (TRO) at each frequency $\boldsymbol{T}(\omega)$ is defined as the self-adjoint matrix $\boldsymbol{T}(\omega)=\boldsymbol{K}^{\dagger}(\omega) \boldsymbol{K}(\omega)$, where ${ }^{\dagger}$ denotes a conjugate transpose (note that time-reversal is equivalent to complex conjugation in the frequency domain). An eigenvalue decomposition of the TRO provides $N$ orthonormal eigenvectors and associated eigenvalues. In the scalar case and for wellresolved point-like targets, each target is associated with one eigenvalue/vector pair: the eigenvalue bears information on the scattering coefficient and the eigenvector bears information on the target's location [8]. Extended targets will, in general, be associated with more than one significant eigenvalue/vector pair depending on their electrical sizes and orientations w.r.t the array. Here, we assume point-like targets; however, under certain conditions, these techniques can be applied to extended targets as well. A detailed analysis on the application of DORT to extended targets classification and imaging was presented in [12].

If the number of array elements $N$ is larger than the number of point-like scatterers $M$, only $M$ eigenvalues will be significant, with the associated eigenvectors spanning the signal subspace. The remaining $N-M$ eigenvectors constitute the null subspace. The DORT method utilizes the signal subspace eigenvectors to construct separate images of each target (selective focusing) [21], [25]. The complex conjugate of the $m^{t h}$ eigenvector (corresponding to the $m^{\text {th }}$ target) is given by

$$
\mathbf{q}_{m}(\omega)=\frac{\mathbf{g}_{m}(\omega)}{\left\|\mathbf{g}_{m}(\omega)\right\|} e^{j \omega \tau}
$$

where $\mathbf{g}_{m}(\omega)$ is the steering vector of location $m$, given by

$$
\mathbf{g}_{m}(\omega)=\left[G_{(m, 1)}(\omega), \ldots, G_{(m, N)}(\omega)\right]^{T}
$$

where $G_{(m, n)}(\omega)$ is the Green's function between the location of target $m$ and the $n^{\text {th }}$ element of the array, and $\left\|\mathbf{g}_{m}\right\|=$ $\sqrt{\left\langle\mathbf{g}_{m}, \mathbf{g}_{m}\right\rangle}$, where $\langle\mathbf{a}, \mathbf{b}\rangle=\mathbf{b}^{\dagger} \mathbf{a}$ denotes inner product, and $\tau$ represents a possible time shift that may result from the lack of precise knowledge about the time reference $t=0$, as discussed before.

To construct the frequency-synthesized image of target $m$, $\mathbf{q}_{m}(\omega)$ is projected onto normalized steering vectors of a synthetic imaging domain. For an arbitrary point $p$ within the imaging domain, this projection is given by

$$
P_{p}^{m}(\omega)=\left\langle\frac{\mathbf{g}_{p}(\omega)}{\left\|\mathbf{g}_{p}(\omega)\right\|}, \mathbf{q}_{m}(\omega)\right\rangle
$$

In the time domain, the projection is computed by taking the inverse Fourier transform of $P_{p}^{m}(\omega)$, that is

$$
P_{p}^{m}(t)=\mathscr{F}^{-1}\left(P_{p}^{m}(\omega)\right)
$$

The focusing time $t_{f}^{m}$ and the focusing location $p_{f}^{m}$ are the instant and location at which the peak of $P_{p}^{m}(t)$ occurs, that is

$$
\left\{p_{f}^{m}, t_{f}^{m}\right\}=\underset{\{p, t\}}{\arg \max }\left(P_{p}^{m}(t)\right)
$$

Finally, the normalized image functional at point $p$ is given as

$$
I_{p}^{m}=\left|\frac{P_{p}^{m}\left(t_{f}^{m}\right)}{\max _{\{p, t\}}\left(P_{p}^{m}(t)\right)}\right|
$$

As an example, consider the imaging of a point-like target located in free-space using a linear TR array of $z$-polarized point sources in a two-dimensional scenario, as illustrated in Fig. 1. The magnitude and phase of the projection at the focusing location in the frequency domain are plotted in Fig. 2. The magnitude is flat over all frequencies and equals to one (maximum value by definition), while the phase behavior is almost linear. This shows that perfect correlation exists between the target eigenvector and the steering vector at the focusing location. Also, in this case, there is a deterministic relationship among frequency components.

The resulting normalized DORT image is shown in Fig. 1. The impact of the interrogating signal bandwidth $B$ (we assume a rectangular spectrum throughout, where $B=f_{\max }-$ $\left.f_{\min }\right)$, and center frequency $f_{c}$ on the image is considered in Fig. 3, where the co- and cross-range profiles of the imaging functional are plotted. Fig. 3(a) shows that by reducing the fractional bandwidth (whether by reducing the bandwidth or 


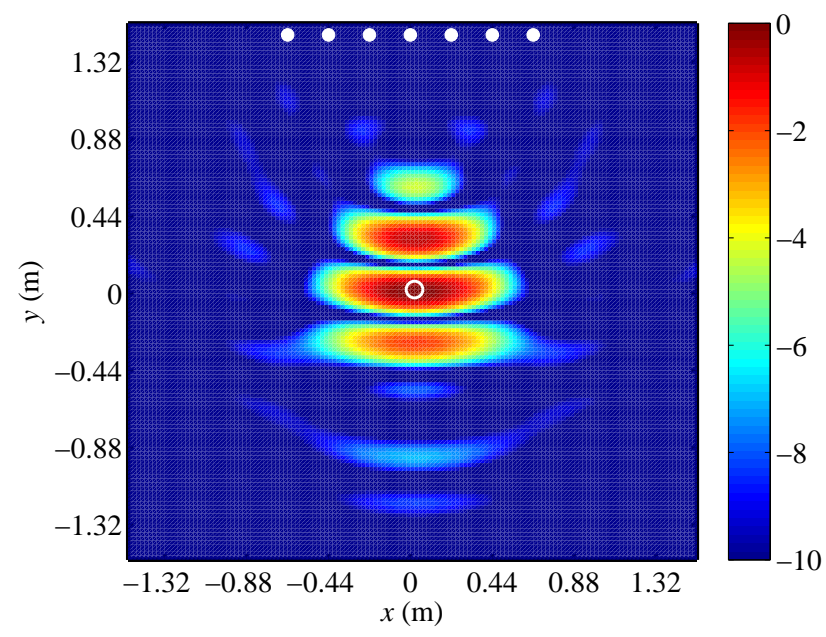

Fig. 1: Normalized DORT image (in $\mathrm{dB}$ scale) of a pointlike target imaged by a linear array. Array elements are indicated by white dots. The target location is indicated by the small white circle. The center frequency is $500 \mathrm{MHz}$ and the bandwidth is $400 \mathrm{MHz}$.

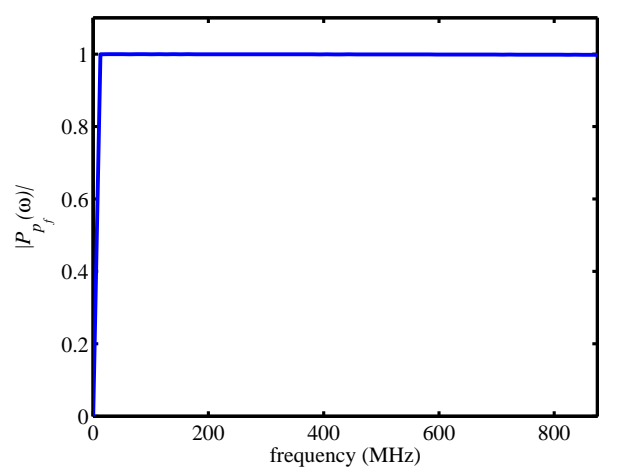

(a)

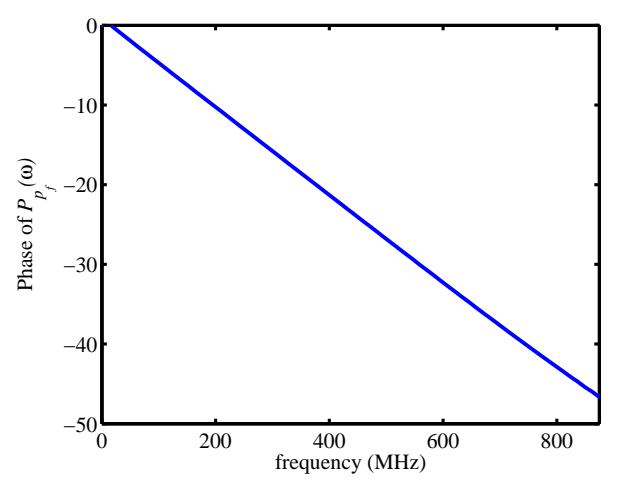

(b)

Fig. 2: Projection (in the frequency domain) of the eigenvector of a target on the imaging domain at the focusing location. The target is point-like and embedded in a uniform medium. (a) Magnitude. (b) Phase.

increasing the center frequency), an increase in the sidelobe level along the co-range results. Increasing the center fre-

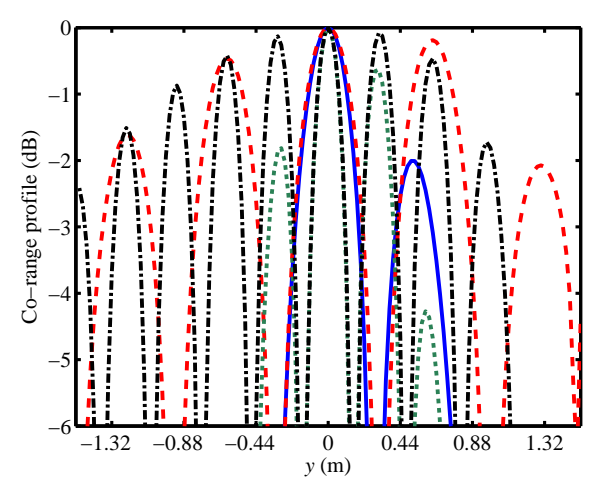

(a)

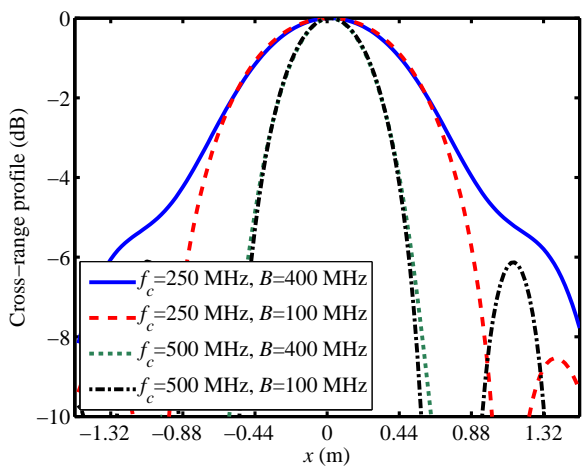

(b)

Fig. 3: Profiles of normalized DORT images produced using four different sets of interrogating signal parameters. (a) Corange. (b) Cross-range.

quency, however, reduces the main lobe width. Note that, for sufficiently far targets imaged by linear arrays, the co-range profile is simply the spatial mapping of the projection $P_{p}(t)$ at the focusing location (as in conventional beamforming). Fig. 3(b) shows that the cross-range profile is hardly affected by the bandwidth. Conversely, it is seen that increasing the center frequency enhances cross-range resolution (from an overall increase on the electrical size of the array), as expected.

\section{B. Frequency-Synthesized MUSIC}

Instead of using the signal subspace as in DORT, MUSIC employs the null subspace to construct a simultaneous image of all targets. The null subspace projection vector at point $p$ $\left(\mathbf{n}_{p}(\omega)\right)$ can be obtained using signal subspace eigenvectors as follows

$$
\mathbf{n}_{p}(\omega)=\frac{\mathbf{g}_{p}(\omega)}{\left\|\mathbf{g}_{p}(\omega)\right\|}-\sum_{m=1}^{M} P_{p}^{m}(\omega) \mathbf{q}_{m}(\omega)
$$

The above equation is illustrated schematically in Fig. 4 (for $N=3$ and one significant eigenvector, that is $M=1$ ). The time domain version of (7) provides $N$ signals that, once transmitted by the array in a time-reversed fashion, produce a beam focusing on $p$ and null fields at each of the $M$ targets locations. For imaging purposes, the norm of $\mathbf{n}_{p}(\omega)$ is first computed and then all frequencies are combined by taking an 


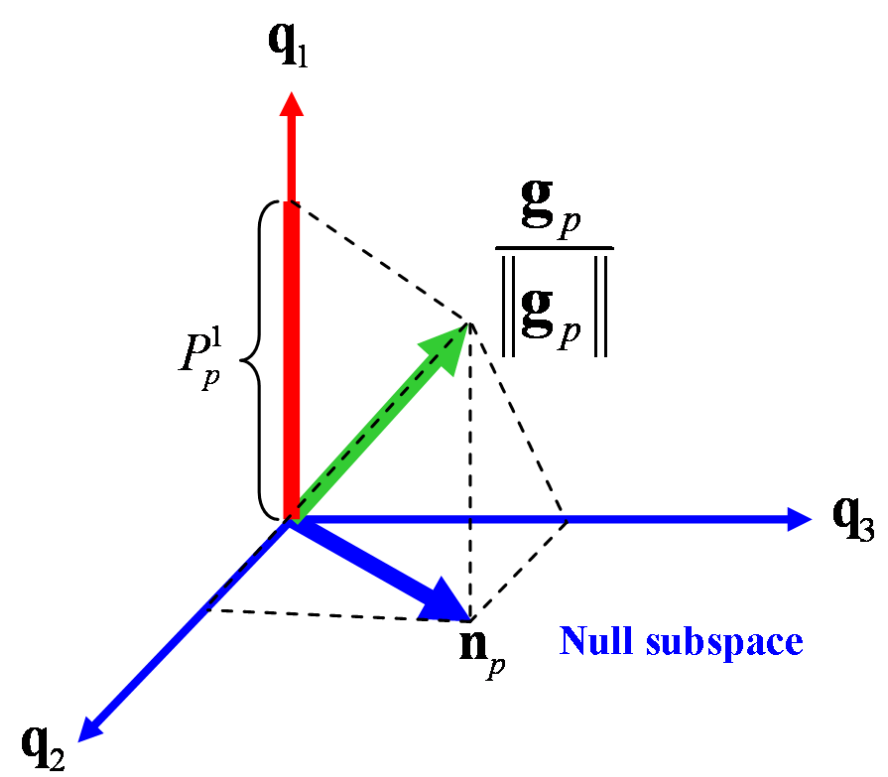

Fig. 4: Diagram illustrating the null subspace projection for the case of three-dimensional space with one significant eigenvector.

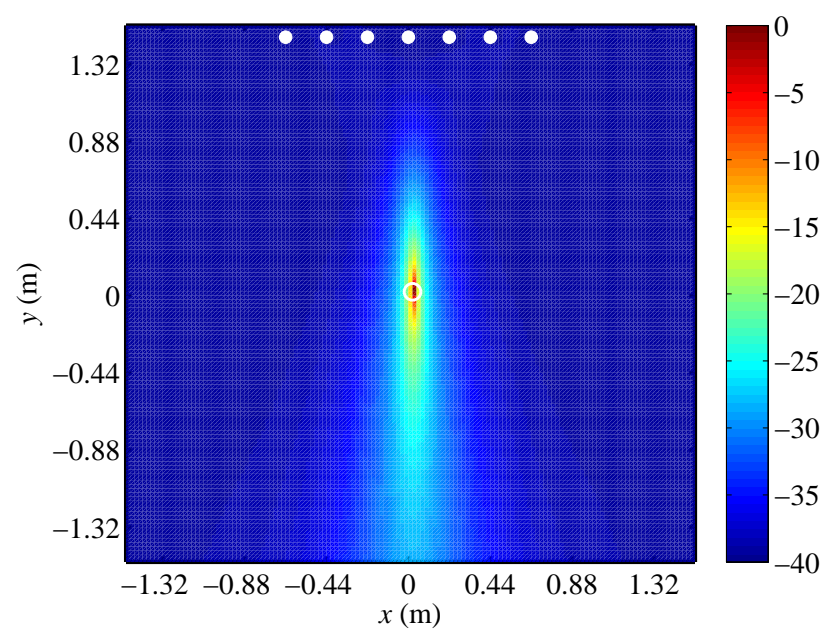

Fig. 5: Same as Fig. 1 using MUSIC.

inverse Fourier transform, yielding a null subspace projection in the time domain $N_{p}(t)$ as follows

$$
N_{p}(t)=\mathscr{F}^{-1}\left(\left\|\mathbf{n}_{p}(\omega)\right\|\right)
$$

All frequency components involved in the above inverse Fourier transform are real; hence, it is irrelevant here to define a focusing time instant. The focusing location is the minimizer of $N_{p}(t)$ which holds for any $t$, so for instance

$$
p_{f}=\underset{p}{\arg \min } N_{p}(t=0)
$$

The MUSIC imaging functional is defined as the reciprocal of the null subspace projection, that is

$$
I_{p}^{M U}=\frac{\min _{p} N_{p}(t=0)}{N_{p}(t=0)}
$$

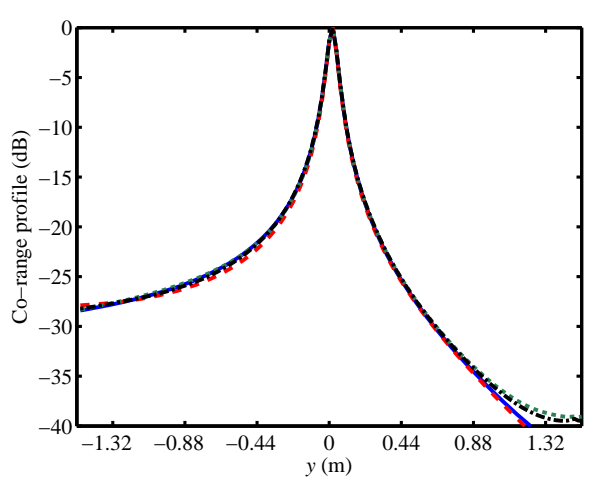

(a)

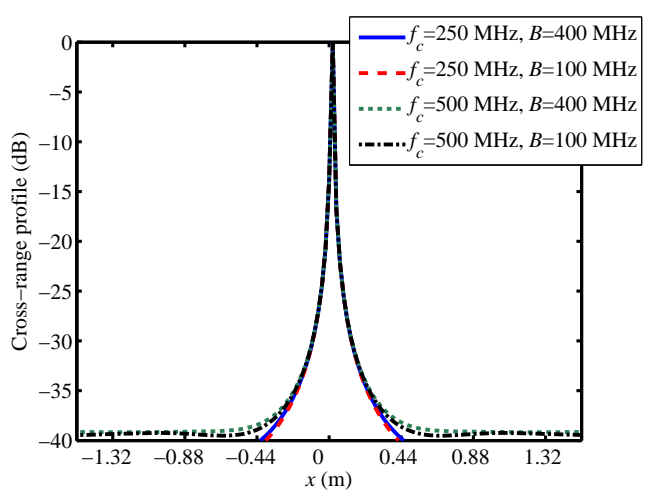

(b)

Fig. 6: Profiles of normalized MUSIC images produced using four different sets of interrogating signal parameters. (a) Corange. (b) Cross-range.

The MUSIC image of the same point-like target considered before is plotted in Fig. 5. The co-range and cross-range profiles for different interrogating signal parameters are shown in Fig. 6. From these plots we observe that, in contrast to the prior DORT images, the (cross-range) resolution of the MUSIC images is mostly independent of the center frequencies and bandwidths considered. However, this is only valid for pointlike targets in uniform media, where UWB operation does not add extra information over that offered by narrowband operation. This will not hold for imaging in random media as will be discussed later on.

\section{Volumetric beamforming: full-aspect DORT and MUSIC}

We next apply the above DORT and MUSIC frequencysynthesized algorithms to a full-aspect (circular) array configuration. The DORT image is shown in Fig. 7. The image profiles along the horizontal direction are shown in Fig. 8. Similarly to the linear array cross-range profile, the full-aspect image resolution is effectively independent of the bandwidth, but is enhanced by increasing the center frequency of operation. Similar to the linear array co-range profile, the full-aspect image has sidelobe levels that increase with a decrease on the fractional bandwidth. However, the sidelobe levels are considerably lower than in the previous linear array case. The full-aspect MUSIC image is shown in Fig. 9. It retains the excellent cross-range resolution of conventional MUSIC along 


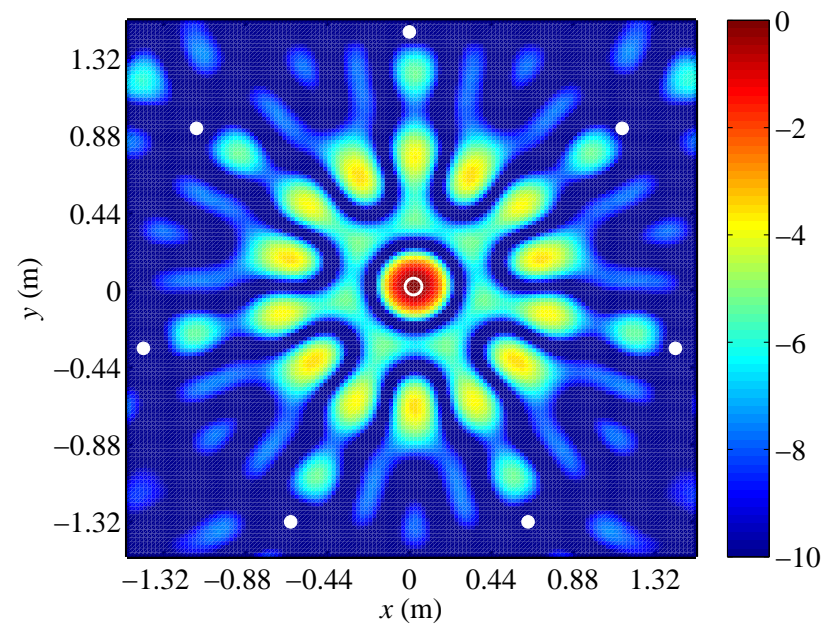

Fig. 7: Same as Fig. 1 using a full-aspect circular array (volumetric DORT).

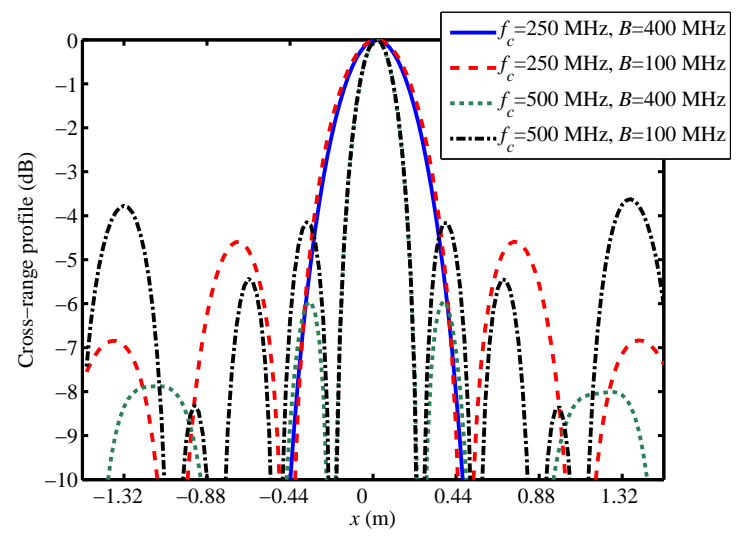

Fig. 8: Normalized volumetric DORT image profiles along $x$ direction produced by using four different sets of interrogating signal parameters.

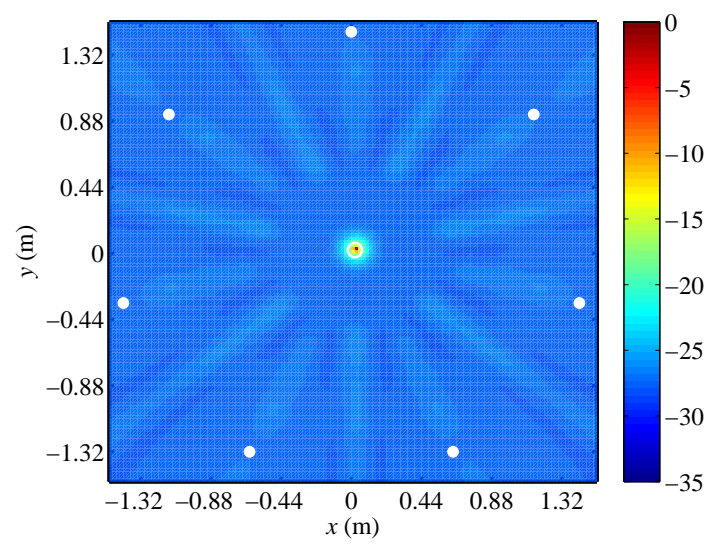

Fig. 9: Same as Fig. 1 using MUSIC and a full-aspect circular array (volumetric MUSIC).

all directions. Such full-aspect imaging can be viewed as a form of "volumetric beamforming".

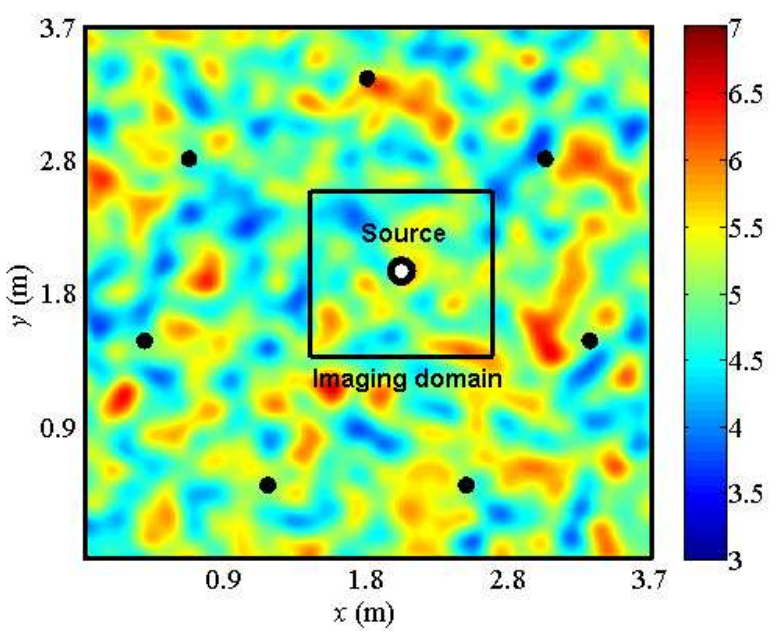

Fig. 10: Problem setup: An active source is embedded in a continuous random medium having the permittivity distribution shown. An array of seven elements is used to record signals emitted by the source and to reconstruct its location within a synthetic imaging domain having the dimensions shown. Imaging is performed assuming a uniform background with permittivity equal to the mean permittivity of the random medium.

\section{Statistical Stability ANALYSis}

\section{A. Theory and approach}

So far, we have considered imaging of targets in uniform (homogeneous) background media. Often times in practice, the exact background distribution is unknown and/or difficult (that is, computationally very costly) to reconstruct. In such cases, the background can be treated as a random medium where there is a mismatch between the actual (physical) background and the synthetic (imaging) domain, each corresponding to a possible (different) realization. In this section, we extend our previous discussion to targets embedded in continuous random media. In this case, the image itself becomes a random variable, whose variance can be used as a measure of statistical stability.

Consider a point-like source (active target) embedded in a random medium realization as shown in Fig. 10. The signal transmitted by the target is recorded by the TR array and used to reconstruct the target location within a synthetic imaging domain with the dimensions shown. The synthetic imaging domain is chosen as a uniform medium with permittivity equal to the mean permittivity of the random medium. The projection at point $p$ in the imaging domain is given by

$$
P_{p}(\omega)=\left\langle\frac{\mathbf{g}_{p}(\omega)}{\left\|\mathbf{g}_{p}(\omega)\right\|}, \frac{\tilde{\mathbf{g}}_{s}(\omega)}{\left\|\tilde{\mathbf{g}}_{s}(\omega)\right\|}\right\rangle
$$

where $\tilde{\mathbf{g}}_{s}(\omega)$ is the steering vector of the source in the random medium, and $\mathbf{g}_{p}(\omega)$ is the steering vector of location $p$ in the (uniform) imaging domain. The mismatch between $\tilde{\mathbf{g}}_{s}(\omega)$ and $\mathbf{g}_{p}(\omega)$ makes $P_{p}(\omega)$ a complex random variable. Its amplitude and phase depend on the random medium realization as shown in Fig. 11. This figure also shows that for a given realization, 


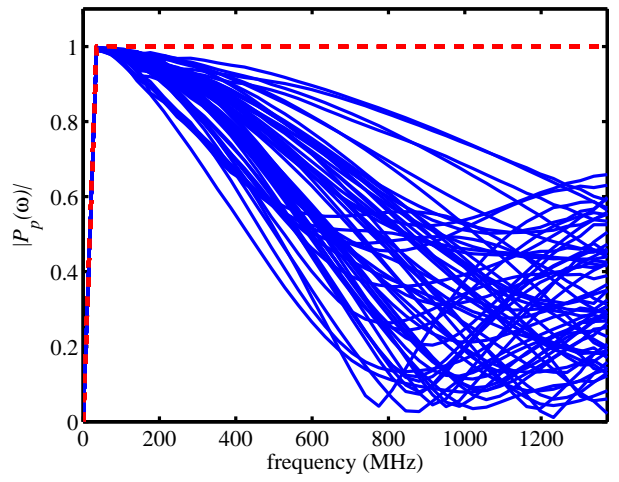

(a)

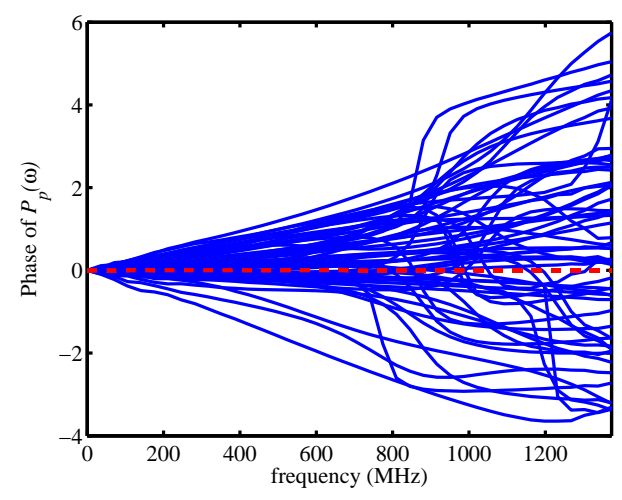

(b)

Fig. 11: Frequency domain projections of the recorded signal vector, from different realizations of the forward problem, at the source location in the imaging domain. (a) Magnitude. (b) Phase. Dotted curves represent the equivalent projections in a known (uniform) background.

the projection is "incoherent", i.e., $P_{p}(\omega)$ has nondeterministic variation with frequency (compare with the dotted curves of the uniform background case).

By decomposing the interrogating signal into $N_{f}$ discrete frequency components, the projection can be assembled into the following complex random vector

$$
\mathbf{p}=\left[P_{p}\left(\omega_{1}\right), \ldots, P_{p}\left(\omega_{N f}\right)\right]^{T}
$$

In the time domain, the projection at $t=0$ (normalized by the number of frequency components to ensure a fair comparison among images produced using different bandwidths) is given by

$$
P_{p}(t=0)=\frac{1}{N_{f}} \sum_{i=1}^{N_{f}}[\mathbf{p}]_{i}
$$

The variance of $P_{p}(t=0)$ can be written as

$$
\operatorname{var}\left(P_{p}(t=0)\right)=\frac{1}{N_{f}^{2}} \sum_{i=1}^{N_{f}} \sum_{j=1}^{N_{f}}[\boldsymbol{C}(\mathbf{p}, \mathbf{p})]_{i j}
$$

where $\boldsymbol{C}(\mathbf{p}, \mathbf{p})$ is the autocovariance matrix of $\mathbf{p}$ (see Appendix). A typical covariance matrix is plotted in Fig. $12\left(l_{c}\right.$, $\sigma, \epsilon_{r m}$ are medium parameters defined in the next section).

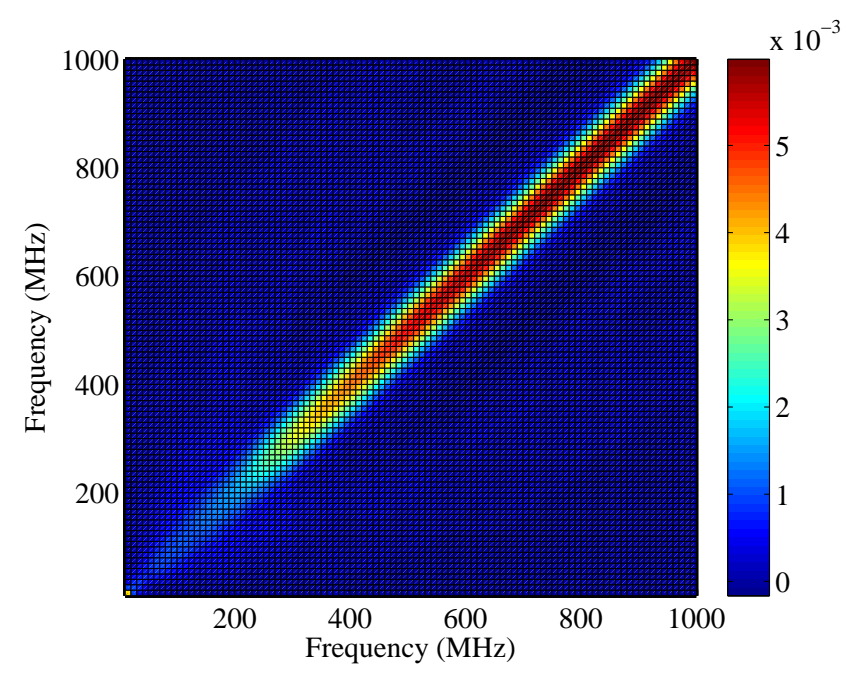

Fig. 12: Covariance matrix of the projection vector for $l_{c}=$ $0.44 \mathrm{~m}, \sigma=0.1 \epsilon_{r m}$, and full-aspect array with radius $R=$ $1.47 \mathrm{~m}$.

The fact that the magnitude of off-diagonal elements is much smaller than that of the diagonal elements is an indication that sufficiently spaced frequency components are decorrelated. In such case, the sum of $N_{f}$ frequency components mimics an ensemble average over $N_{f}$ (uncorrelated) realizations. As a result, the image variance should decay asymptotically with the bandwidth as $\simeq 1 / N_{f}$ under the assumption that the $N_{f}$ components have equal variance.

\section{B. Simulation results}

In this section, we use the average (over $p$ ) in (14) as a measure of stability. We will call it simply the image variance. We carry out a parametric study on the effect of different signal and medium parameters on the image stability. We consider both the image variance itself and its fractional decrease with bandwidth. The latter is used as a measure of frequency decorrelation.

The background medium in the forward problem is a realization of a continuous random permittivity with a clipped Gaussian distribution and a Gaussian spatial correlation function [27]. This distribution is characterized by three parameters (see Fig. 10): the mean permittivity $\epsilon_{r m}$, the standard deviation of the permittivity $\sigma$, and the spatial correlation length $l_{c}$ [27], [28]. For this analysis, we choose $\epsilon_{r m}=5$, and compute the image variance using Monte Carlo technique by ensemble averaging over one hundred realizations. Note that in practice, one has access to only one realization of the random medium and hence only one image; nevertheless, this image variance sets an upper bound on the deviation of the image from the (stable) ensemble average according to the Chebyshev inequality [15]. All simulations are carried out using the finitedifference time-domain method [29].

1) Interrogating signal parameters: Fig. 13 shows the image average and variance for narrowband and wideband operations around the same center frequency $f_{c}$. It is clear from this figure that the narrowband image is unstable: the 
image variance is large and spreads out throughout the imaging domain. On the contrary, the wideband images are stable: their variance is small and confined to a small region around the true target location.

For a given frequency band, the image variance can be computed for all possible $f_{c}, B$ combinations within that band, as illustrated in Fig. 14. This figure shows that for a given $B$, the variance increases with larger $f_{c}$. This is because higher frequency components are better able to resolve the random medium fluctuations for the present choice of $l_{c}$.

2) Random medium parameters: The dependence of the image variance on the correlation length $l_{c}$ for different $B$ and $f_{c}$ is illustrated in Fig. 15(a) and (b), respectively. We can distinguish two main regimes. The first regime consists of $l_{c} \ll \lambda_{c}$, where the random medium effectively behaves as a uniform medium with (effective) permittivity $\epsilon_{r m}$. As $l_{c}$ increases, the signal is gradually more affected by the medium fluctuations and consequently the variance increases. The second regime consists of $l_{c} \geq \lambda_{c}$. In this case, the variance is large but increases only slightly with $l_{c}$. For $l_{c} \gg \lambda_{c}$, the medium behaves again as a uniform medium (at least locally in the imaging domain), but this time with random permittivity that varies from one realization to the other. Note also that, as seen from Fig. 15(b), the transition between these two regimes occurs at shorter correlation lengths when $f_{c}$ increases, as expected.

The variance we have computed so far corresponds to images captured at $t=0$. However, when there is any mismatch between the average permittivity of the actual background and that of the imaging domain, $t=0$ is no longer the optimal focusing instant, as we discussed before. This is typical in the limit of long $l_{c}$. Perhaps a more accurate measure of stability is therefore to consider the variance of the null subspace projection $N_{p}(t=0)$, that is, the reciprocal of the MUSIC imaging functional. MUSIC would provide target's image at the same location as DORT image had the latter been captured at the optimal focusing instant. The counterpart of Fig. 15 using the null subspace variance measure is shown in Fig. 16. The main difference is that, in the long $l_{c}$ regime, the variance decreases monotonically with $l_{c}$. This shows that MUSIC images are more robust to permittivity mismatch.

3) Array geometry: We next illustrate the effect of the TR array geometry on the image variance. For full-aspect circular arrays, reducing the array radius $R$ decreases the two-way travel path of the electromagnetic signal; therefore, the image variance decreases as shown in Fig. 17(a). The use of a linear array with same number of elements and (nearest) distance $L=R$ from the source, increases the image variance significantly. In addition, the image variance becomes less sensitive to bandwidth increase, as evident by the fractional decrease in the image variance seen in Fig. 17(b). The covariance matrices of full-aspect array and linear array images are plotted in Fig. 18, for comparison. It is clear that the frequency correlation in the linear array case is much stronger than in the full-aspect case. This is due to the fact that a larger region of the domain is "spanned" by the interrogating field using a full-aspect geometry as opposed to a linear-aspect geometry.

\section{CONCLUSION}

We have presented a new frequency-synthesized technique for constructing ultrawideband images using time-reversal DORT and MUSIC imaging. The proposed technique does not require backpropagation of time-reversed waves in the imaging domain. Once the steering vectors (Green's function) of the imaging domain are computed and stored, they can be reused to construct images of different targets in the same domain, for different interrogating signal parameters (central frequency and bandwidth). It was shown that, for point-like targets in a uniform background medium, the imaged target location is effectively invariant with respect to the range of interrogating signal parameters considered. In addition, the resolution of time-reversal MUSIC images is effectively invariant with respect to the signal parameters as well. It was also observed that when using full-aspect arrays, the resolution of time-reversal DORT images is hardly affected by the bandwidths considered here. In the case of random medium backgrounds, the image can be considered as a random functional itself, whose variance is related to the covariance matrix of the constituting frequency components. The more pronounced is the frequency decorrelation in the random medium, the faster is the decrease of the image variance with increasing bandwidth. A Monte Carlo study of the statistical stability of the resulting images was carried out under different combinations of interrogating signal parameters, random medium parameters, and sensor array geometries. This work can be further extended to analyze the statistical stability of differential TR [12] techniques and to examine scenarios involving time-varying background media.

\section{APPENDIX}

PROOF OF (14)

The mean square of $P_{p}(t=0)$ is given by

$$
E\left[P_{p}^{2}(t=0)\right]=\frac{1}{N_{f}^{2}} \sum_{i=1}^{N_{f}} \sum_{j=1}^{N_{f}}[\boldsymbol{R}(\mathbf{p}, \mathbf{p})]_{i j}
$$

where $\boldsymbol{R}(\mathbf{p}, \mathbf{p})=E\left[\mathbf{p p}^{\dagger}\right]$ is the autocorrelation matrix of $\mathbf{p}$ and $E[$.$] denotes expected value. The matrix \boldsymbol{R}(\mathbf{p}, \mathbf{p})$ can be expanded as

$$
\boldsymbol{R}(\mathbf{p}, \mathbf{p})=E[\mathbf{p}] E\left[\mathbf{p}^{\dagger}\right]+\boldsymbol{C}(\mathbf{p}, \mathbf{p})
$$

where $\boldsymbol{C}(\mathbf{p}, \mathbf{p})$ is the autocovariance matrix of $\mathbf{p}$ [30]. Substituting (16) in (15) yields

$E\left[P_{p}^{2}(t=0)\right]=\frac{1}{N_{f}^{2}} \sum_{i=1}^{N_{f}} \sum_{j=1}^{N_{f}} E[\mathbf{p}]_{i} E\left[\mathbf{p}^{\dagger}\right]_{j}+\frac{1}{N_{f}^{2}} \sum_{i=1}^{N_{f}} \sum_{j=1}^{N_{f}}[\boldsymbol{C}(\mathbf{p}, \mathbf{p})]_{i j}$

The first term in the right hand side is $E\left[P_{p}(t=0)\right]^{2}$, so the variance of $P_{p}(t=0)$ is given by (14).

\section{ACKNOWLEDGMENTS}

This work has been supported by the National Science Foundation (NSF) under grant ECCS-0925272 and by the Ohio Supercomputing Center (OSC) under grant PAS-0110. 


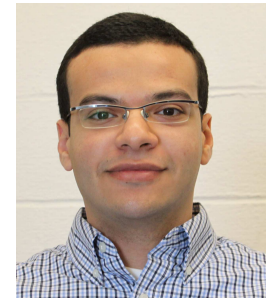

Ahmed E. Fouda was born in Cairo in 1985 . He received his B.Sc. (with honors) degree in electrical engineering from Ain Shams University, Cairo, Egypt, in 2007, and the M.Sc. from the same university in 2010. His M.Sc. research involved applying metamaterials concepts in designing enhancedperformance RF components.

He worked as a teaching and research assistant at Ain Shams University for two years. In 2009, he joined the ElectroScience Lab. at the Ohio State University as a graduate research associate where he is currently working towards his Ph.D. His current research interests include electromagnetic time-reversal, radar signal processing, inverse problems, and computational electromagnetics. Mr. Fouda is the recipient of the SBC Presidential Fellowship from The Ohio State University and the IEEE MTT-S Graduate Fellowship for 2012.

Fernando L. Teixeira received the B.S. and M.S degrees in Electrical Engineering from the Pontifical Catholic University of Rio de Janeiro (PUC-Rio), Brazil, in 1991 and 1995, respectively, and the Ph.D degree in Electrical Engineering from the University of Illinois at Urbana-Champaign, in 1999. From 1999 to 2000 he was a Postdoctoral Associate with the Massachusetts Institute of Technology. In 2000 he joined the The Ohio State University, where he is now a Professor with the Department of Electrical and Computer Engineering and also affiliated with

the ElectroScience Laboratory.

His current research interests include ultrawideband inverse scattering, electromagnetic sensors for hydrocarbon exploration, metamaterials, and computational electromagnetics. He has published over 100 journal papers and has lectured extensively in these areas. Dr. Teixeira currently serves as Associate Editor for the IEEE Antennas and Wireless Propagation Letters. He has received a number of awards for this research, including the CAREER Award from the National Science Foundation, the triennial Booker Fellowship from the US Committee of the International Union of Radio Science (USNC/URSI), the Outstanding Young Engineer Award from the IEEE Microwave Society (MTT-S), and the Harrison Award for Faculty Excellence from the Ohio State University.

\section{REFERENCES}

[1] M. Fink, "Time reversal mirrors," J. Phys. D.: Appl. Phys., vol. 26, pp. 1333-1350, 1993.

[2] F. Wu, J. L. Thomas, and M. Fink, "Time reversal of ultrasonic fieldspart ii: Experimental results," IEEE Trans. Ultrason., Ferroelectr., Freq. Control, vol. 39, pp. 567-578, September 1992.

[3] H. T. Nguyen, J. B. Andersen, G. F. Pedersen, P. Kyritsi, and P. C. F. Eggers, "Time reversal in wireless communications: A measurementbased investigation," IEEE Trans. Wireless Commun., vol. 5, pp. 22422252, 2006.

[4] C. J. Leuschen and R. G. Plumb, "A matched-filter-based reverse-time migration algorithm for ground-penetrating radar data," IEEE Trans. Geosci. Remote Sensing, vol. 39, pp. 1257-1264, 2006.

[5] F. Foroozan and A. Asif, "Time-reversal ground-penetrating radar: Range estimation with Cramer-Rao lower bounds," IEEE Trans. Geosci. Remote Sensing, vol. 48, pp. 3698 -3708, 2010.

[6] L. Li, W. Zhang, and F. Li, "A novel autofocusing approach for realtime through-wall imaging under unknown wall characteristics," IEEE Trans. Geosci. Remote Sensing, vol. 48, pp. 423 - 431, 2010.

[7] M. E. Yavuz and F. L. Teixeira, "Space-frequency ultrawideband timereversal imaging," IEEE Trans. Geosci. Remote Sensing, vol. 46, pp. 1115-1124, 2008.

[8] - "On the sensitivity of time-reversal imaging techniques to model perturbations," IEEE Trans. Antennas Propagat., vol. 56, pp. 834-843, 2008

[9] E. A. Marengo, F. K. Gruber, and F. Simonetti, "Time-reversal MUSIC imaging of extended targets," IEEE Trans. Image Processing, vol. 16, pp. 1967-1984, 2007.

[10] P. Kosmas and C. M. Rappaport, "Time reversal with the FDTD method for microwave breast cancer detection," IEEE Trans. Microwave Theory Tech., vol. 53, pp. 2317-2323, 2005.

[11] J. M. F. Moura and Y. W. Jin, "Time reversal imaging by adaptive interference canceling," IEEE Trans. Signal Process., vol. 56, pp. $233-$ 247, 2008.

[12] A. E. Fouda and F. L. Teixeira, "Imaging and tracking of targets in clutter using differential time-reversal techniques," Waves in Random and Complex Media, vol. 22, pp. 66-108, 2012.

[13] A. Devaney, "Super-resolution processing of multistatic data using time reversal and MUSIC," Accessed in Nov. 2009, unpublished paper, preprint available on the authors web site. Available Online: http://www.ece.neu. edu/faculty/devaney/.

[14] P. Blomgren, G. Papanicolaou, C. Tsogka, and J. Berryman, "Super resolution in time-reversal acoustics," J. Acoust. Soc. Am., vol. 111, pp. 230-248, 2002.

[15] G. Papanicolaou, L. Ryzhik, and K. Solna, "Statistical stability in time reversal," SIAM J. Appl. Math., vol. 64, pp. 1133-55, 2004.

[16] A. Derode, A. Tourin, and M. Fink, "Random multiple scattering of ultrasound. ii. is time reversal a self-averaging process?" Phys. Rev. E, vol. 64,2001

[17] J. G. Berryman, L. Borcea, G. Papanicolaou, and C. Tsogka, "Statistically stable ultrasonic imaging in random media," J. Acoust. Soc. Am., vol. 112, pp. 1509-1522, 2002.

[18] L. Borcea, G. Papanicolaou, C. Tsogka, and J. Berryman, "Imaging and time reversal in random media," Inverse Prob., vol. 18, pp. 1247-1279, 2002.

[19] D. Liu, S. Vasudevan, J. Krolik, G. Bal, and L. Carin, "Electromagnetic time-reversal source localization in changing media: Experiment and analysis," IEEE Trans. Antennas Propagat., vol. 55, pp. 344-354, 2007.

[20] D. Liu, J. Krolik, and L. Carin, "Electromagnetic target detection in uncertain media: Time-reversal and minimum-variance algorithms," IEEE Trans. Geosci. Remote Sensing, vol. 45, pp. 934 - 944, 2007.

[21] C. Prada and M. Fink, "Eigenmodes of the time reversal operator: A solution to selective focusing in multiple-target media," Wave Motion, vol. 20, pp. 151-163, 1994.

[22] C. Prada, S. Mannevile, D. Spoliansky, and M. Fink, "Decomposition of the time reversal operator: Detection and selective focusing on two scatterers," J. Acoust. Soc. Am., vol. 99, pp. 2067-2076, 1996.

[23] H. Tortel, G. Micolau, and M. Saillard, "Decomposition of the time reversal operator for electromagnetic scattering," J. Electromagn. Waves Appl., vol. 13, pp. 687-719, 1999.

[24] G. Micolau, M. Saillard, and P. Borderies, "DORT method as applied to ultrawideband signals for detection of buried objects," IEEE Trans. Geosci. Remote Sens., vol. 41, pp. 1813-1820, 2003.

[25] M. E. Yavuz and F. L. Teixeira, "Full time-domain DORT for ultrawideband fields in dispersive, random inhomogeneous media," IEEE Trans. Antennas Propagat., vol. 54, pp. 2305-2315, 2006. 
[26] A. J. Devaney, "Time reversal imaging of obscured targets from multistatic data," IEEE Trans. Antennas Propagat., vol. 53, pp. 1600-1610, 2005.

[27] F. L. Teixeira, W. C. Chew, M. Straka, M. L. Oristaglio, and T. Wang, "Finite-difference time-domain simulation of ground penetrating radar on dispersive, inhomogeneous and conductive soils," IEEE Trans. Geosci. Remote Sensing, vol. 36, pp. 1928-1937, 1998.

[28] C. D. Moss, F. L. Teixeira, Y. E. Yang, and J. A. Kong, "Finite-difference time-domain simulation of scattering from objects in continuous random media," IEEE Trans. Geosci. Remote Sens., vol. 40, pp. 178-186, 2002.

[29] A. Taflove and S. Hagness, Computational Electrodynamics: The FiniteDifference Time-Domain Method, 3rd ed. Norwood, MA: Artech House, 2005.

[30] A. Papoulis and S. U. Pillai, Probability, Random variables and Stochastic Processes, 4th ed. New York: McGraw-Hill, 2002.

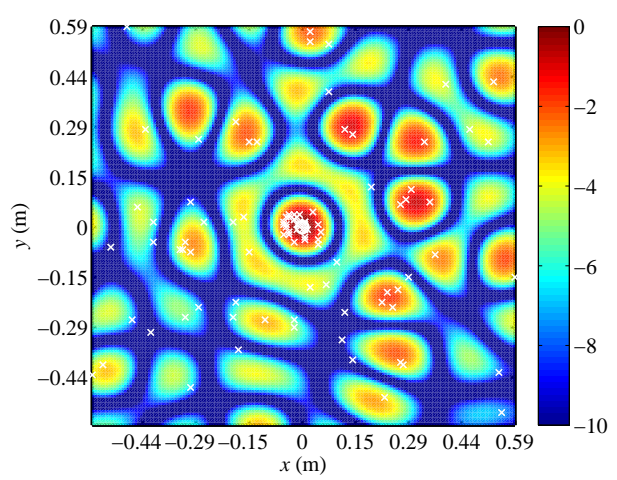

(a)

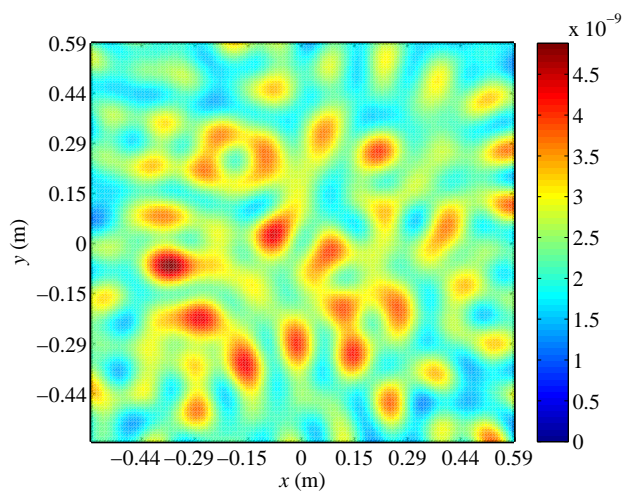

(b)

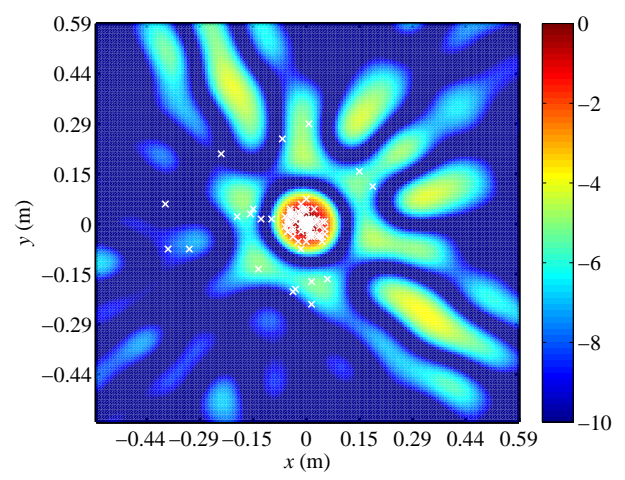

(c)

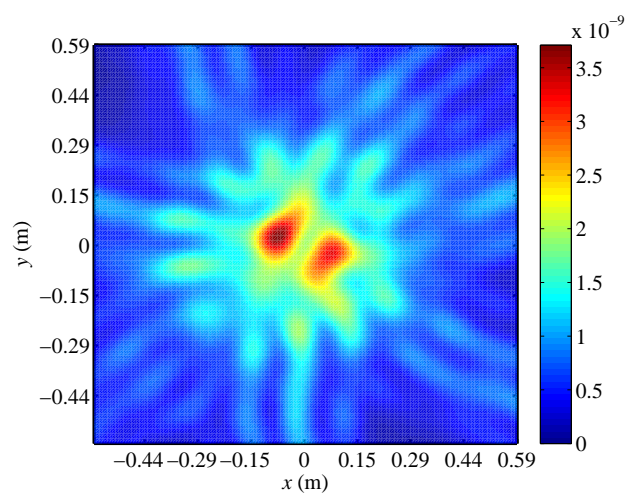

(d)

Fig. 13: Beamforming results for $f_{c}=500 \mathrm{MHz}$ and different bandwidths. (a) Image average for $B=100 \mathrm{MHz}$. Actual source location is indicated by ' $\mathrm{o}$ '. Estimated source locations from different realizations are indicated by 'x's. The r.m.s. error in the source localization is $35 \mathrm{~cm}$. (b) Image variance for $B=100 \mathrm{MHz}$. (c) Image average for $B=400 \mathrm{MHz}$. The r.m.s. error in source localization is $11 \mathrm{~cm}$. (d) Image variance for $B=400 \mathrm{MHz}$. 


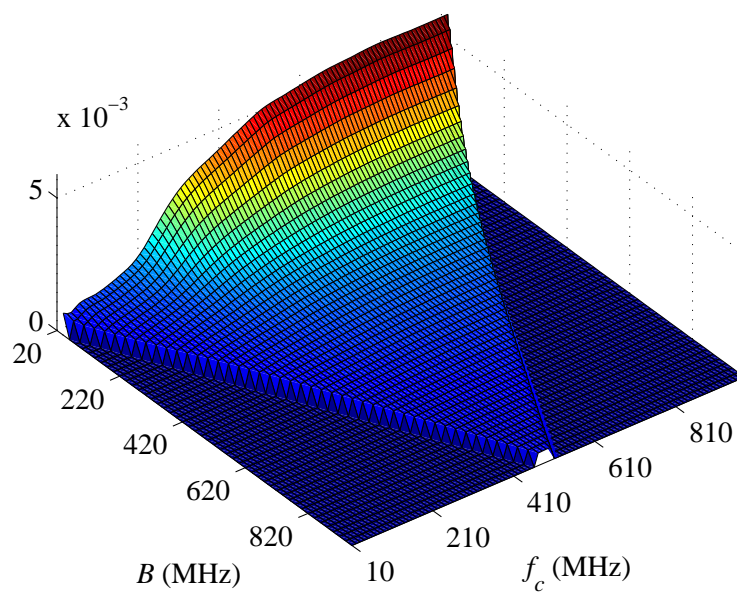

Fig. 14: Image variance versus bandwidth and center frequency, for $l_{c}=0.44 \mathrm{~m}$.

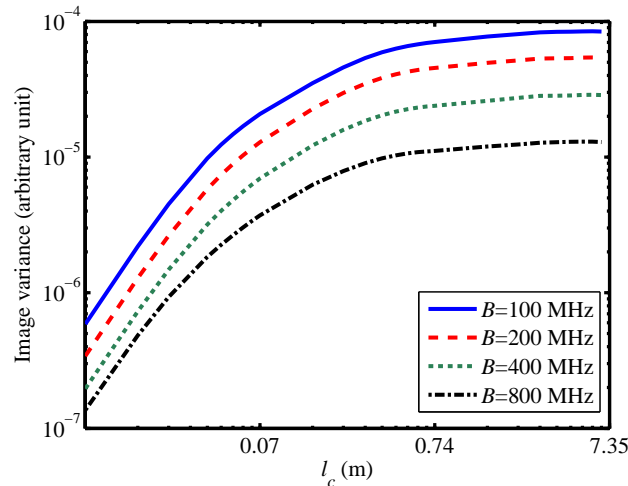

(a)

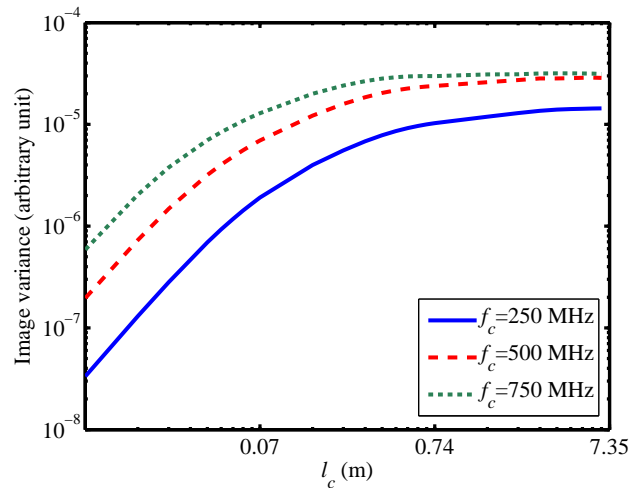

(b)

Fig. 15: Image variance versus correlation length $l_{c}$ for (a) $f_{c}=500 \mathrm{MHz}$ and varying bandwidth $B$, and (b) for $B=400$ $\mathrm{MHz}$ and varying center frequency $f_{c}$.

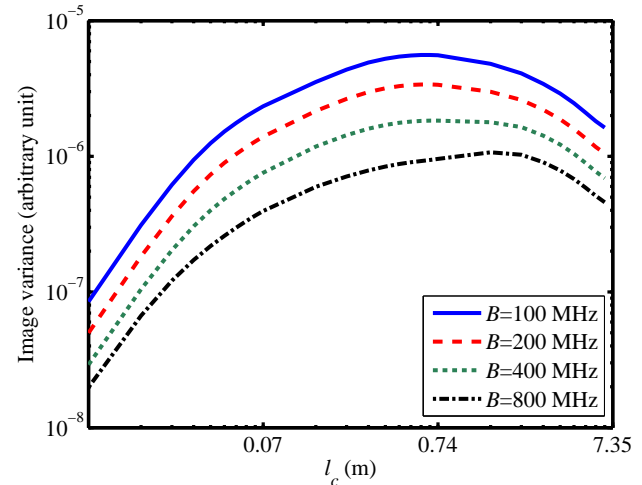

(a)

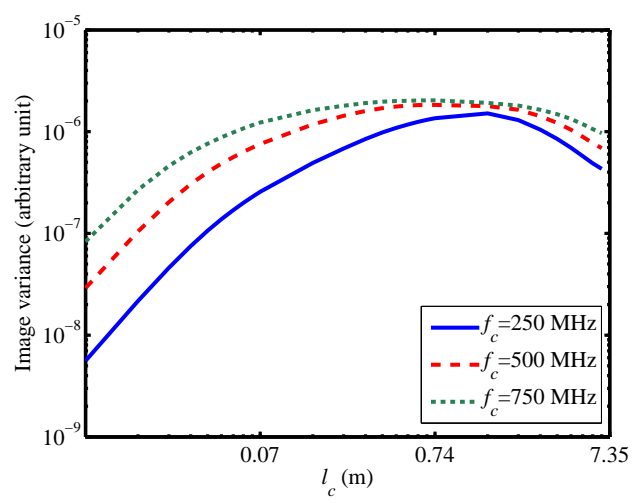

(b)

Fig. 16: Same as Fig. 15 using MUSIC. 


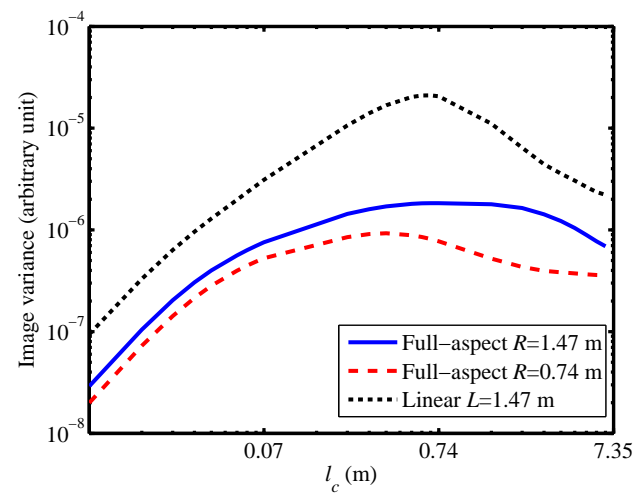

(a)

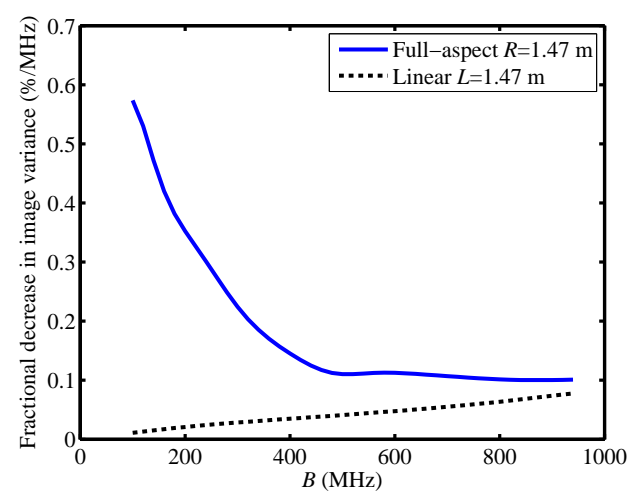

(b)

Fig. 17: Effect of array geometry. (a) Image variance versus correlation length for $f_{c}=500 \mathrm{MHz}, B=400 \mathrm{MHz}$. and three array geometries. (b) Fractional decrease in image variance with bandwidth for $l_{c}=2.94 \mathrm{~m}$ and $f_{c}=500 \mathrm{MHz}$.

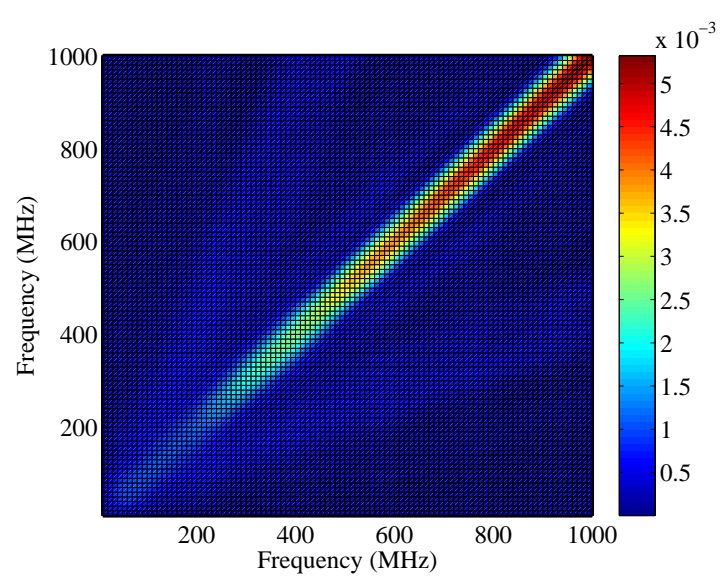

(a)

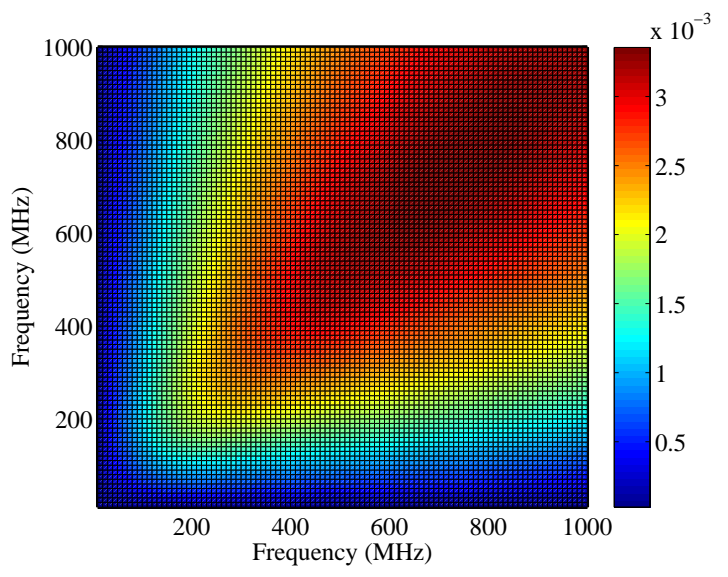

(b)

Fig. 18: Covariance matrices for $l_{c}=2.94 \mathrm{~m}$, using (a) fullaspect array and (b) limited-aspect array configurations. 